\title{
Droplet Size Spatial Distribution Model of Liquid Jets Injected into Subsonic Crossflow
}

\author{
Luhao Liu, ${ }^{1}$ Lijun Yang $\mathbb{D}^{1,2}$ and Qingfei Fu ${ }^{1,2}$ \\ ${ }^{1}$ School of Astronautics, Beijing University of Aeronautics and Astronautics, 100083, China \\ ${ }^{2}$ Beijing Advanced Innovation Center for Big Data-Based Precision Medicine, Beihang University, 100083, China \\ Correspondence should be addressed to Lijun Yang; yanglijun@buaa.edu.cn
}

Received 24 December 2019; Revised 20 May 2020; Accepted 25 June 2020; Published 17 July 2020

Academic Editor: Wei Lin

Copyright (c) 2020 Luhao Liu et al. This is an open access article distributed under the Creative Commons Attribution License, which permits unrestricted use, distribution, and reproduction in any medium, provided the original work is properly cited.

\begin{abstract}
Liquid jet injected into transverse subsonic gaseous flow has been widely utilized in many industrial applications. It is useful to determine the spatial distribution of generated droplets in the near-field region for high-efficiency combustion. In this paper, we propose a simplified model to predict droplet spatial distribution in transverse subsonic gaseous flow. Linear stability analysis has been used to determine the disturbance growth rate on the surface of a liquid column. When the amplitude of disturbance is of the same order of magnitude as jet radius, the liquid jet breaks up into ligaments. We can make an assumption that the generation rate of small droplet equals to liquid breakup rates, which varies with a spatial location under this circumstance. Combining these relations with the definition of SMD (Sauter mean diameter), a semitheoretical relation to evaluate droplet spatial distribution along the liquid column can be established. The present model has been compared with empirical relation based on experiments under different conditions. Results indicate that in the surface breakup region, the current model shows great consistency with experimental observations while there exists a relatively large discrepancy between the current model and experimental observation in the column breakup region because of its strong nonlinear effect near the breakup point. In addition, the effects of flow parameters on droplet size spatial distribution have been investigated.
\end{abstract}

\section{Introduction}

Liquid jet injected into a transverse gaseous flow is widely used in many industrial applications like scramjet in hypersonic vehicles [1-6]. Compared with the traditional injection method, the transverse injection system has many advantages such as rapid atomization and relatively simple structure. In general, atomization processes can be divided into two parts which are primary breakup and secondary breakup, respectively. The present paper concentrates on the characteristics of the primary breakup of liquid injected into a transverse gaseous flow.

In this research field, an earlier study focuses on liquid jets exposed to supersonic crossflow [7,8]. When a liquid jet is in a high-speed transverse gaseous flow, an aerodynamic force stimulates a strong disturbing wave on the liquid surface. The breakup process is quite rapid because of the high gaseous speed. In their experiments, high frequency, large amplitude waves controlled by an aerodynamic force were observed. When the airstream Mach number is large enough, the liquid column goes through a strong atomization process that disturbing wave cannot be observed on the jet surface. In order to solve this problem, Xiao et al. [9], and Li et al. [10], utilized high-quality numerical simulation to investigate primary breakup processes of a liquid jet exposed into supersonic crossflow, which agrees well with experimental results. In addition, under supersonic airflow condition, it generates a detached bow shock wave before the injection point [11], which transforms supersonic airstream into the subsonic airstream. Therefore, research focus has shifted to subsonic transverse gaseous crossflow in the last few decades.

For nonturbulent round jet injected into gaseous crossflow, there existed numerous empirical graphs to classify the primary breakup regime. It can be found that $\mathrm{We}_{G}$ (gaseous Weber number) is the most commonly used parameter to define breakup regime $[12,13]$. Wu et al. [14] firstly used $\mathrm{We}_{G}-q$ ( $q$ is the liquid-gas momentum ratio) map to classify the primary breakup regime, which can be mainly divided 
into two parts: column breakup regime and surface breakup regime. Sallam et al. [15] used $\mathrm{We}_{g}-\left(\lambda_{s} / d_{j}\right)$ map to show the correlation between $\lambda_{s} / d_{j}$ and breakup regime where $\lambda_{s}$ is the wavelength of instability waves developing on the windward side of a liquid jet. It could be found that surface wavelength decreases with the increase of gas Weber number.

Recently, many investigators studied the trajectory of a liquid column, penetration height, and distribution of droplet with advanced experimental equipment. Rezaei et al. [16] used laser sheet planar imaging technology to get instantaneous spatial spray images and obtained the correlation of primary breakup length with different fuel temperatures in a pressure swirl injector. Amighi and Ashgriz [17] provided plenty of experimental data of atomization processes and determined the correlation between trajectories of water jet injected into subsonic crossflow air and different flow parameters. In addition, they utilized the laser light sheet illumination technique and shadowgraphy to establish a relation between global droplet size and various parameters like jet velocity, air temperature, and injector diameter [18]. Broumand and Birouk [19] summarized these correlations between breakup distance or height of a liquid jet exposed to subsonic gaseous crossflow and various physical parameters.

It is well known that a liquid jet goes through a series of atomization processes and finally became a group of tiny droplets. Hence, it is quite significant for a subsequent combustion process to determine the distribution of Sauter mean diameter (SMD) in a flow field. Nevertheless, the existed SMD prediction methods mainly concentrate on empirical correlations related to experimental data [20]. It is quite obvious that these correlations depend strongly on experimental conditions, which cannot explain the physical nature hidden in related breakup phenomenons. Therefore, some theoretical models have been established to evaluate SMD. For instance, Varga et al. [21], developed a dual-instability mode to explain the breakup process of liquid film exposed to a high-speed gaseous flow, which agreed well with experiments.

It should be noticed that in the flow field, the value of SMD measured by the Malvern system in the experiment is full-field SMD distribution which is not precise enough for further optimum design. Therefore, droplet size spatial distribution which means SMD is a function of spatial locations is significant for deeper investigation. Unfortunately, there has rarely related research about how to determine SMD spatial distribution by a theoretical or semitheoretical method, which has a great meaning of optimum design for a nextgeneration engine.

In this paper, we utilize linear stability analysis based on full-wavelength assumption to obtain a surface instability wave growth rate, and then, it can be combined with the nonturbulent round liquid jet breakup rate observed by Sallam et al. [15] in experiments. A semitheoretical spatial SMD distribution prediction model can be determined in the nearfield region. This prediction model coincides quite well to the present empirical correlation obtained by plenty of experiments in the surface breakup region. In addition, the effect of flow parameters like injection velocity and transverse gaseous velocity on droplet size spatial distribution will be investigated as well.

\section{The Droplet Size Spatial Distribution Model}

Many papers have justified that unstable wave exists on the surface of the liquid column [22, 23]. The initial unstable wave propagates along the liquid jet direction while strong interaction between transverse gas and liquid column makes the amplitude of unstable wave larger. In order to simplify the present problem, a series of assumptions have been made as follows. The transverse gaseous flow is incompressible and irrotational. According to Amini [23], jet bending and deformation processes are negligible when the gas-liquid momentum ratio is not sufficiently high; therefore, liquid jet preserves its cylindrical shape before the breakup point. In addition, the geometry effect of the injector and gravity force has been neglected. The initial stage of breakup process is schematically showed in Figure 1, where $u_{G}$ denotes the velocity of transverse gaseous flow, $u_{L}$ is the velocity of the liquid jet, $d_{j}$ is the initial diameter of the liquid column, which is equal to the diameter of an injector in the present analysis, and $\lambda$ is the wavelength of surface instability wave.

There has an assumption that unstable wave within one wavelength will be shed from a cylindrical surface by an aerodynamic force and then form as a small ligament, i.e.,

$$
D_{\text {lig }} \propto \lambda
$$

The scale of primary droplets formed at the tip of small ligaments is given by the following expression, i.e.,

$$
D_{\text {pri }}=\beta D_{\text {lig }}
$$

where $D_{\text {pri }}$ is the diameter of droplets formed by a primary breakup, $D_{\text {lig }}$ is the diameter of ligaments, and $\beta$ is the constant related to form small droplets in a regime of Rayleigh breakup which equals to 1.2 suggested by Sallam et al. [15].

When a liquid jet is injected into a transverse gaseous flow, a tangential aerodynamic force around a liquid column makes ligaments detached from the liquid column. There exists an assumption that ligaments shedding from the interface of a liquid jet per unit wavelength equal to a liquid breakup rate which is a small droplet generation rate as well. Therefore, we can establish the mass conservation relation, i.e.,

$$
m_{\text {rate }}(\lambda) d \lambda \propto \rho_{l} D_{\text {pri }}^{3} n_{\text {rate }}(\lambda) d \lambda,
$$

where $n_{\text {rate }}(\lambda)$ represents the formation number of small droplets per unit wavelength of a column surface. The mass flow rate induced by surface instability wave per unit wavelength is proportional to reduced shedding velocity, i.e., $\lambda / \tau$ , where $\tau$ is the characteristic time that instability wave reaches sufficient amplitude compared with the radius of a liquid column (Mayer [24]). It can be related to the growth rate of instability wave on the liquid surface caused by 


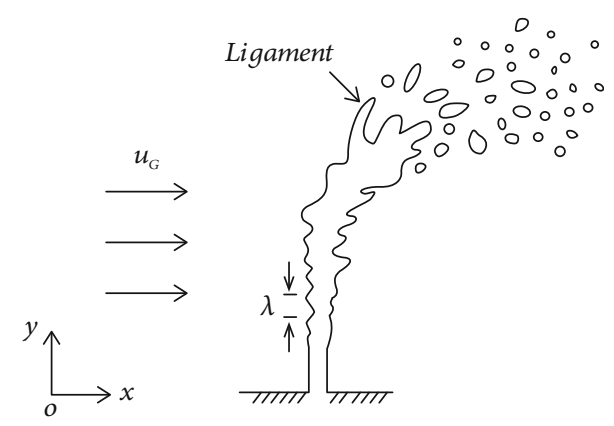

FIGURE 1: Schematic diagram of a liquid jet in transverse crossflow.

transverse airflow. Hence, the characteristic time is inversely proportional to the instability growth rate of surface wave, i.e.,

$$
\tau \propto \frac{1}{\omega_{R}},
$$

where $\omega_{R}$ is the growth rate of instability wave. Therefore, the mass flow rate of shedding surface waves per unit wavelength gives

$$
m_{\text {rate }}(\lambda) d \lambda \propto \rho_{L} \lambda \omega_{R} d \lambda
$$

Combining Equations (3) and (5), we can obtain

$$
D_{\text {pri }}^{2} n_{\text {rate }}(\lambda) d \lambda \propto \frac{\lambda \omega_{R}}{D_{\text {pri }}} d \lambda
$$

By integrating Equation (6) from minimum to maximum wavelength of surface instability wave and then substituting Equations (1) and (2) into Equation (6), we can obtain

$$
\int_{\lambda_{\min }}^{\lambda_{\max }} D_{\text {pri }}^{2} n_{\text {rate }}(\lambda) d \lambda \propto \int_{k_{\min }}^{k_{\max }} \frac{2 \pi}{\beta} \frac{\omega_{R}}{k^{2}} d k
$$

It is clear that the definition of Sauter mean diameter is

$$
\operatorname{SMD}=\frac{\int_{\lambda_{\min }}^{\lambda_{\max }} D_{\text {pri }}{ }^{3} n_{\text {rate }}(\lambda) d \lambda}{\int_{\lambda_{\min }}^{\lambda_{\max }} D_{\text {pri }}{ }^{2} n_{\text {rate }}(\lambda) d \lambda} .
$$

Substituting Equations (3) and (7) into Equation (8), we can obtain

$$
\operatorname{SMD}(y)=C \frac{\beta}{2 \pi} \frac{m_{\text {rate }}(y)}{\int_{k_{\min }}^{k_{\max }}\left(\omega_{R} / k^{2}\right) d k},
$$

where $k_{\min }$ and $k_{\max }$ are the minimum and maximum wave number, respectively. Because previous relationship such as Equation (1) to Equation (7) is not a strict equation, it is necessary to introduce an integral constant $C$, which depends on the structure of injector and physical properties of fluids. When we settle down the value of $C$, it cannot be changed under different flow rates [25]. $m_{\text {rate }}$ is the liquid breakup rate, for round nonturbulent liquid jets injected into a transverse gaseous flow; $m_{\text {rate }}$ can be expressed as follows (Sallam et al. [15]):

$$
m_{\text {rate }}(y)=6.89 \times 10^{-4} \rho_{L} u_{P} \exp \left(\frac{5.43 y}{y_{b}}\right),
$$

where $u_{P}$ is the liquid droplet velocity flowing in the transverse crossflow that can be expressed as

$$
u_{P}=6.7 u_{\infty} \sqrt{\frac{\rho_{G}}{\rho_{L}}},
$$

where $u_{\infty}$ is the transverse gaseous velocity and $y_{b}$ is the breakup height of the liquid column, which can be expressed as $y_{b}=8.05 q^{0.5} D_{0}$ where $D_{0}$ is the diameter of the injector. We should notice that it depends on various parameters such as liquid-gas momentum ratio, injector diameter, and Ohnesorge number. The expressions of $y_{b}$ under different circumstances can be found in Broumand and Birouk [19]. It should be noticed that on the upstream side of the liquid jet, an aerodynamic force tends to accelerate gas toward the liquid jet in transverse stream direction near the upstream stagnation point, which makes liquid column compressed and develops Rayleigh-Taylor wave on the surface of the liquid column.

Wang et al. [22] utilized the linear instability analysis to obtain dispersion relation of surface waves on the round nonturbulent liquid jets injected into transverse subsonic gaseous airflow. Therefore, we can make some necessary simplifications and dispersion equation reduces to

$$
\omega_{R}^{2}=\frac{\left(k u_{L}\right)^{2}}{1+\bar{\rho}}-\frac{\left(k u_{L}\right)^{2}}{(1+\bar{\rho})^{2}}-\frac{\sigma k^{3}}{\rho_{L}(1+\bar{\rho})}+\frac{k \rho_{G} u_{G}^{2}}{2 h(1+\bar{\rho}) \rho_{L}},
$$

where $a$ is the radius of the liquid jet, $h=a / 8$ is the reduced thickness because of the compressive effect of an aerodynamic force, $\bar{\rho}=\rho_{G} / \rho_{L}$ is the density ratio, and $\sigma$ is the surface tension.

In the expression of SMD, the range of integration needs to be determined before calculation. $k_{\min }$ corresponds to the minimum value of unstable wavenumber; before the breakup of the liquid jet, surface wave develops noticeably and its wavelength is assumed to be equal to the diameter of the injector. After that point, the liquid column would not hold its cylindrical shape and breakup into ligaments and large droplets. Therefore, the minimum wavenumber can be expressed as follows, i.e.,

$$
k_{\min }=\frac{2 \pi}{d_{j}} .
$$

Subsequently, we should determine the value of $k_{\max }$. We set $\omega_{R}=0$ and obtain the cutoff wavenumber. However, in order to make sure the convergence of calculation, we should set minimum wavelength equals to $0.1 d_{j}$ according to Ashgriz and Mashayek [26]. Therefore, the maximum wavenumber in the present calculation is 


$$
k_{\max }=\frac{2 \pi}{0.1 d_{j}} .
$$

During the theoretical derivation, the relation between primary droplet and ligaments has been established by Ray- leigh breakup. Then, the total number of primary droplets can be connected with the liquid breakup rate measuring by existed experiments. Finally, we can substitute Equations (10) and (12) into Equation (9); then, a semitheoretical relation based on linear stability analysis can be expressed as follows, i.e.,

$$
\operatorname{SMD}(y)=C \frac{\beta}{2 \pi} \exp \left(\frac{5.43 y}{y_{b}}\right) \times \frac{6.89 \times 10^{-4} \rho_{L} u_{P}}{\int_{k_{\min }}^{k_{\max }} \sqrt{\left(u_{L}^{2} /\left(k^{2}(1+\bar{\rho})\right)\right)-\left(u_{L}^{2} /\left(k^{2}(1+\bar{\rho})^{2}\right)\right)-\left(\sigma /\left(\rho_{L} k(1+\bar{\rho})\right)\right)+\left(\rho_{G} u_{G}^{2} /\left(2 k^{3} h(1+\bar{\rho}) \rho_{L}\right)\right)} d k} .
$$

In reality, the precise primary breakup phenomenon of liquid jet injected into a transverse gaseous flow includes column breakup, which characteristics are quite different from a surface breakup. To be more specific, within the column breakup regime, the liquid column breakups to large ligaments entirely and the cylindrical shape of the liquid jet would not be maintained. More significantly, surface instability wave will disappear and linear instability analysis will not adjust to the present condition. Therefore, the related phenomenon considered and discussed in this paper just simplifies some breakup processes and obtains SMD spatial distribution in the surface breakup regime near the exit of the injector.

\section{Results and Discussion}

In this section, we will first verify the correction and application scope of the present model and then investigate the effect of different flow parameters on the maximum growth rate of surface instability wave and spatial distribution of droplet size in a transverse gaseous flow.

3.1. Verification of Model. In order to verify the validness of the present semitheoretical relation, experimental conditions in Kihm et al. [27] are utilized as basic parameters. Water was injected through an injector which diameter equals to $0.5 \mathrm{~mm}$. Other conditions have presented here, which includes three different transverse gas Reynolds number of 50000, 60000, and 70000, respectively. The air-liquid ratio has been set as 25,30 , and 35 , and the liquid flow rate varies between 1.58 and $4.21 \mathrm{ml} / \mathrm{s}$. In addition, in their experiments, the value of SMD has been measured by the Malvern detector at the $x$ location of $10 \mathrm{~mm}$ and $20 \mathrm{~mm}$ downstream from the center of the injector exit.

Based on these experimental results, an empirical relation of SMD spatial distribution can be obtained by the Buckingham-PI theorem, which is shown as follows:

$\frac{\mathrm{SMD}}{D_{0}}=1.015 \times 10^{19} \mathrm{Re}_{g}^{-3.5998} \mathrm{Re}_{f}^{-1.8094} \mathrm{We}_{g}^{2.2474}\left(\frac{x}{D_{0}}\right)^{-0.6867}\left(\frac{y}{D_{0}}\right)^{1.9718}$, where $D_{0}$ is the diameter of the injector, $\mathrm{We}_{g}$ is the gas Weber number, and $\mathrm{Re}_{g}$ and $\mathrm{Re}_{f}$ are gas Reynolds number and liquid Reynolds number, respectively. $x$ is the horizontal distance between breakup point and injector exit while $y$ is the vertical distance between them. Because the liquid breakup rate is independent of the horizontal direction, we can measure the value of SMD at breakup point, i.e., $x=x_{b}$. The correlation of liquid column breakup distance with injector diameter can be found in Broumand and Birouk [19], which has been shown previously. We choose four groups of different flow rates in order to compare it with our present model.

According to experimental observations, breakup time can be calculated by

$$
t_{b}=\frac{y_{b}}{v_{j}}
$$

where $y_{b}$ is the breakup height and $v_{j}$ is the liquid injection velocity. Results show that regardless of injection velocity, breakup time is about $3 \mathrm{~ms}$. It indicates that the existence time of liquid column in crossflow is quite short and the surface instability wave development process is quite rapid. The comparison of SMD spatial distribution between the present model and an empirical relation is shown in Figure 2.

It should be noticed that the unit of $x$-coordinate is $y / D_{0}$ and the unit of $y$-coordinate is $\mathrm{SMD} / D_{0}$, which indicates the relative size between breakup height or SMD and diameter of injector, respectively. It shows that the overall tendency of SMD increases synchronously with the development of liquid column. Because limited injectors and flow rates were tested in experiments of Kihm et al. [27], the integral constant $C$ is set to an optimum value, i.e., $C=3 \times 10^{-6}$ according to calculation result and previous attempts. The changes in temperature and pressure of transverse airflow are reflected in physical properties like gas density, which do not vary noticeably in experiments. According to the experimental conditions, we set temperature and pressure of airflow as constant in our analysis. 


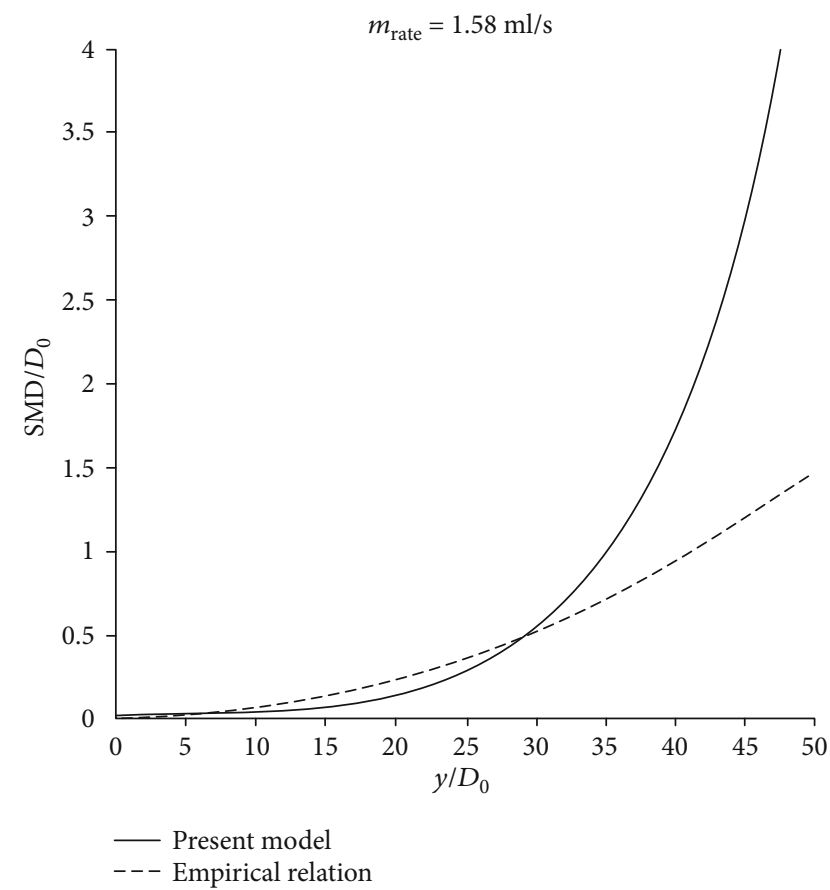

(a)

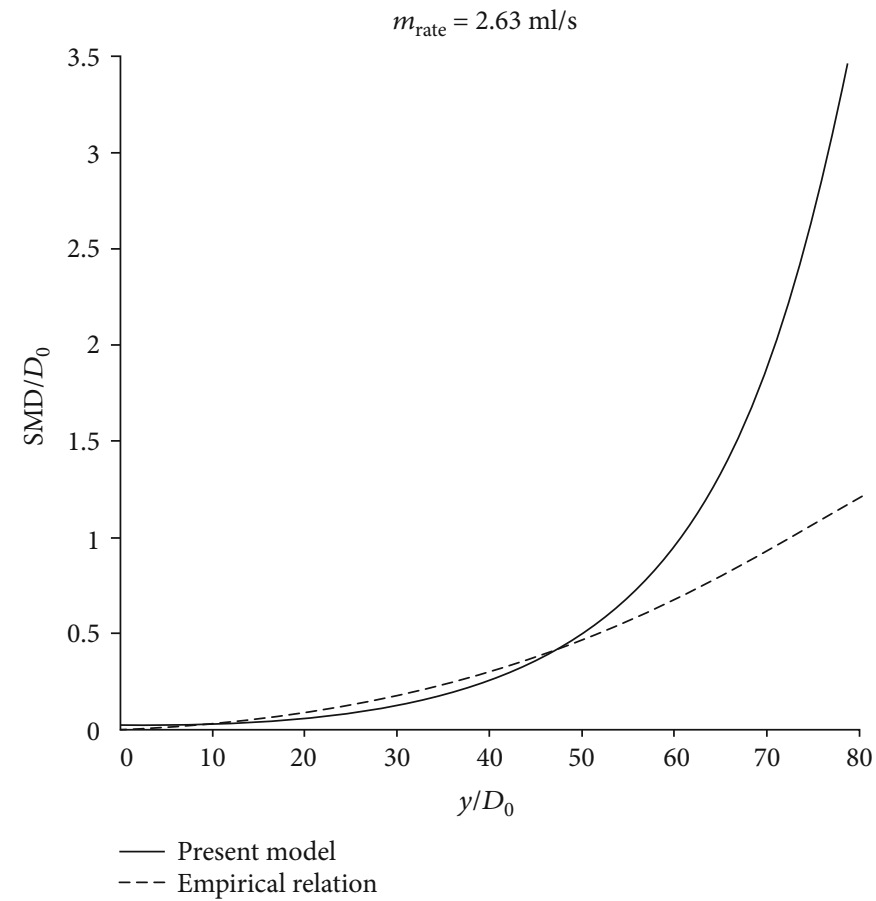

(b)

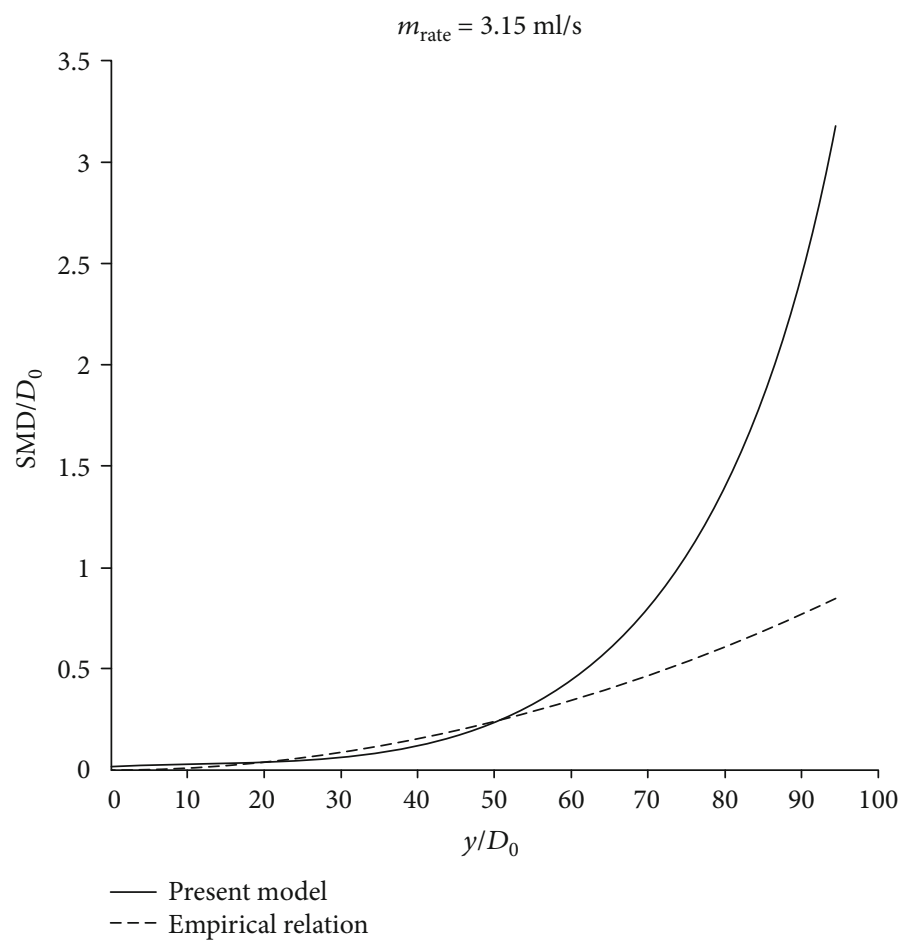

(c)

Figure 2: Continued. 


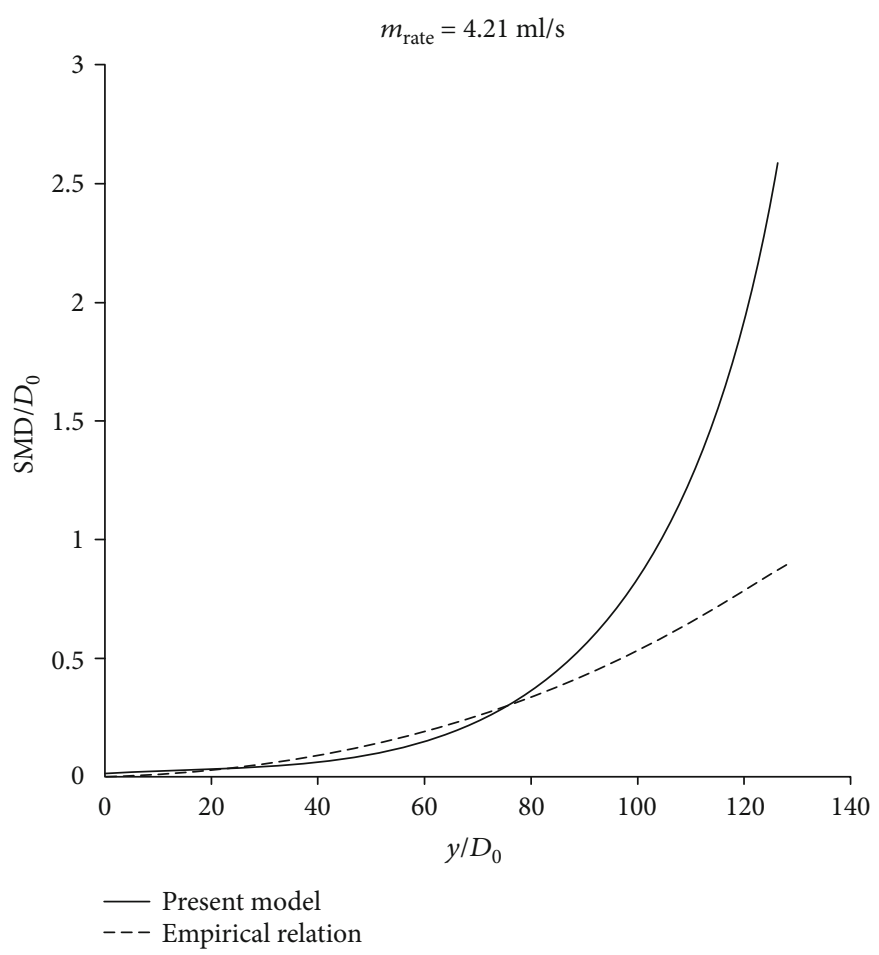

(d)

FIGURE 2: Comparison of the SMD spatial distribution between present model predictions and empirical relation by Kihm et al. [27]. (a) $m_{\text {rate }}=1.58 \mathrm{ml} / \mathrm{s}$. (b) $m_{\text {rate }}=2.63 \mathrm{ml} / \mathrm{s}$. (c) $m_{\text {rate }}=3.15 \mathrm{ml} / \mathrm{s}$. (d) $m_{\text {rate }}=4.21 \mathrm{ml} / \mathrm{s}$.

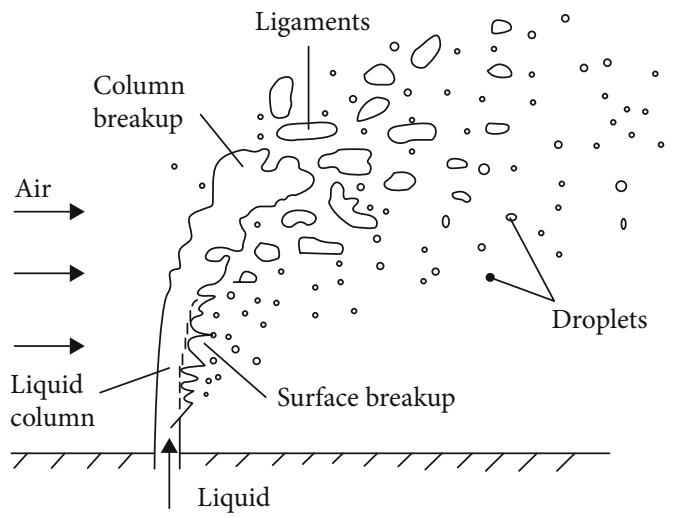

FIGURE 3: Schematic diagram of the breakup region of liquid jet injected into transverse airflow.

It is straightforward that breakup height increases monotonously with the increase of liquid flow rate. As shown in Figure 2 that small droplets are generated after leaving the exit of the injector which is the effect of tangential force acting on the surface of the liquid column. In the present model, it indicates that for short-wavelength disturbance near the exit of the injector, it produces relatively small droplets and droplet size spatial distribution predicted by the present semitheoretical model coincides quite well with empirical relation; the deviation between empirical relation and present model is small in surface breakup region. In addition, at the same height under different liquid flow rates, it indicates that SMD decreases with the increase of liquid flow rate. It means that surface breakup intensifies with the increase of liquid-gas momentum ratio, which results in smaller SMD correspondingly. This tendency has also been observed by Tambe et al. [28], in their experiments. According to what we have mentioned above, it justifies the correctness of the present model in the surface breakup region.

Nevertheless, the increase of the liquid-gas momentum ratio can also change the breakup region, i.e., from the surface breakup region to the column breakup region. As shown in Figure 3 [14], in the column breakup region, liquid column and surface wave do not exist and liquid column breakups into large ligaments near the breakup point. We should notice that the present model is based on linear stability analysis and the development process of surface wave existed on a liquid column. When it approaches the breakup point, present simplified dispersion Equation (12) is not acceptable because of its strong nonlinear effect and the disappearance of surface waves. Therefore, the present model has a relatively large discrepancy with empirical relations in the column breakup region. In order to obtain more comprehensive and precise data, we can refer to some high-quality simulation such as Liu et al. [29], to understand the spray characteristics or breakup mechanism of a liquid jet in crossflow.

3.2. Effect of Flow Parameters. Flow parameters have significant effects on the instability characteristics and breakup processes of a liquid jet in a transverse gaseous flow. In the present investigation, we set the gas-liquid momentum ratio as a constant which confines breakup length and breakup 


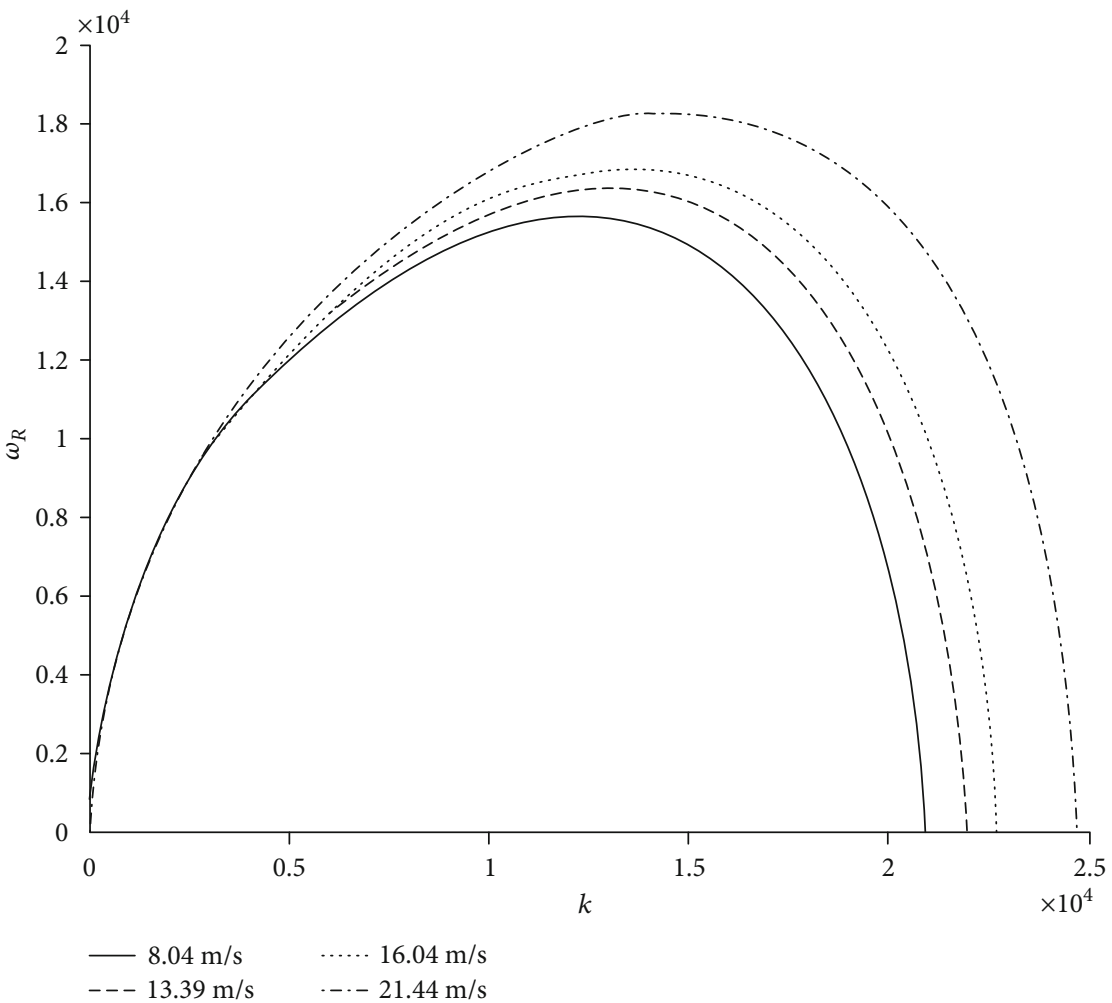

(a)

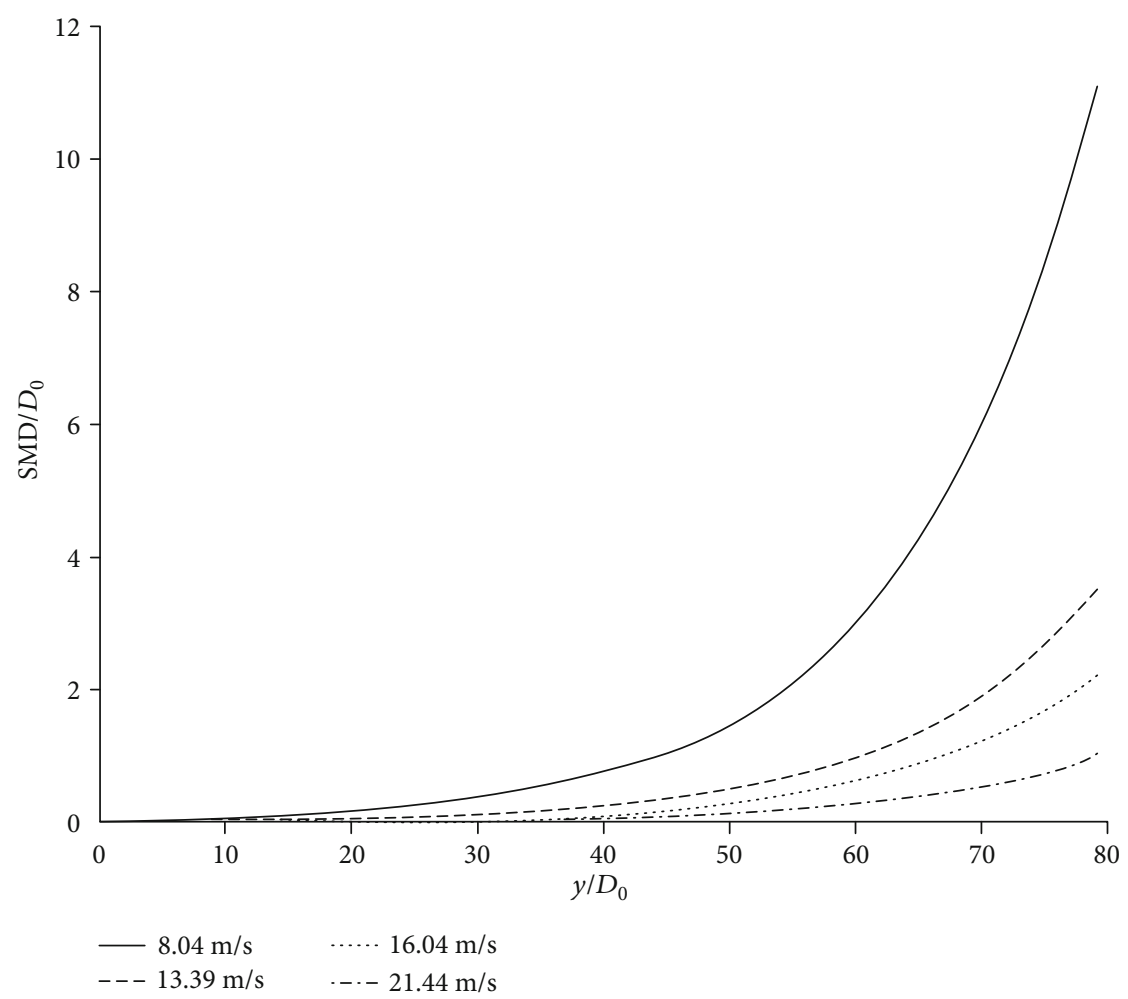

(b)

FiguRE 4: Effect of injection velocity to (a) surface wave growth rate and (b) droplet size distribution $\left(\sigma=0.0728 \mathrm{~N} / \mathrm{m}^{2}, \rho_{G}=1.29 \mathrm{~kg} / \mathrm{m}^{3}\right.$, $\rho_{L}=1000 \mathrm{~kg} / \mathrm{m}^{3}$, and $\left.q=96.35\right)$. 
mode which are the same under different conditions and changes other flow parameters like injection velocity, liquid density, transverse gaseous velocity, and density to investigate the variation trends of maximum growth rate and cutoff wavenumber of surface instability wave. In addition, by changing flow parameters, we can obtain the droplet spatial distribution curve under various conditions. Through this way, we can predict SMD spatial distribution and its variation tendency with different flow parameters as well. The size distribution of droplets can be evaluated by comparing the value of SMD at the same height in the effective range of the present model.

3.2.1. Effect of Injection Velocity. The injection velocity is related to injector geometry, liquid mass flow rate, and pressure drop of the injector. The maximum growth rate and droplet distribution under different injection velocities are shown in Figure 4.

It should be noticed that these four injection velocities are not set randomly. These velocities correspond to the mass flow rate of the injector in related experiments. As shown in Figure 4(a), the maximum growth rate and cutoff wavenumber increase with the increase of injection velocity, which indicates that the liquid column is easier to break up and the wavelength of surface instability wave is smaller with larger injection velocity. These have been verified by previous researchers [23]. As shown in Figure 4(b), the droplet size distribution shows that SMD will be smaller for larger injection velocity. Physically, it can be explained that with larger injection velocity, smaller wavelength generated at the onset of liquid column shrinks into smaller droplets according to Rayleigh breakup mechanism. Furthermore, for larger injection velocity, the development of disturbing wave is insufficient, which also generates smaller droplets.

3.2.2. Effect of Liquid Density. Liquid density is a physical property which is independent to the injector. The effect of liquid density on the maximum growth rate and droplet distribution is shown in Figure 5.

It shows that in Figure 5(a), the maximum growth rate increases with the increase of liquid density while cutoff wavenumber rarely varies with liquid density, which means that initial wavelength on column surface is the same for different kinds of liquids. In Figure 5(b), it shows that SMD increases with denser liquid. It can be explained that the expression of droplet size distribution is inverse proportional to liquid density. And denser liquid refers to a smaller maximum growth rate that makes SMD larger. Hence, the value of SMD at specified height increases with the increase of liquid density, correspondingly.

3.2.3. Effect of Transverse Gaseous Velocity. Transverse gaseous velocity is related to the upstream condition. The effect of transverse gaseous velocity is shown in Figure 6.

Similar to Figure 4, these four transverse gaseous velocities refer to the corresponding gaseous Reynolds number in experiments. It shows in Figure 6(a) that increasing transverse gaseous flow promotes the wave development process on the surface of the liquid column. And the wavelength of the surface wave decreases with larger gaseous velocity, which can be represented by cutoff wavenumber. It indicates that in Figure 6(b) larger gaseous velocity makes SMD smaller. In physics, it can be explained that increasing gaseous velocity enlarges the aerodynamic force that could promote instability of jet, which has been observed in experiments by Behzad et al. [30]. Furthermore, similar to the effect of injection velocity, Rayleigh breakup would occur near the injector exit. It forms smaller droplets at the onset of the liquid column with larger gaseous velocity and smaller wavelength of surface wave.

3.2.4. Effect of Transverse Gaseous Density. The transverse gaseous pressure can be represented by gaseous density that pressure of transverse flow increases with gaseous density. The effect of transverse gaseous density is presented in Figure 7.

It shows in Figure 7(a) that different from the effect liquid density, the increase of gas density makes the maximum growth rate and cutoff wavenumber larger, which means denser gas induces a stronger aerodynamic force on the surface of the liquid column and promotes the breakup of the liquid jet. It can be indicated in Figure 7(b) that in a high pressure environment, denser gas suppresses the occurrence of column breakup and generation process of ligaments, which makes the value of SMD smaller. It is verified by high precision simulations [31].

3.2.5. Effect of Surface Tension. Surface tension is a physical parameter which is related to temperature and pressure. In the present investigation, we assume that surface tension does not vary with other parameters and only changes the value of surface tension to explore its impact. The effect of surface tension is presented in Figure 8.

It shows in Figure 8(a) that the increase of surface tension makes maximum growth rate and cutoff wavenumber smaller, which means surface tension has an effect to keep the cylindrical shape of the liquid jet, which restrains the breakup process. As shown in Figure $8(\mathrm{~b})$, the value of SMD enlarges with the increase of surface tension. It can be explained that surface tension makes the maximum growth rate smaller and surface tension term is located in the denominator of expression of SMD spatial distribution. Therefore, increasing surface tension makes SMD larger.

\section{Conclusion}

This paper established a semitheoretical model to predict droplet size spatial distribution along the liquid column in the surface breakup region of liquid jet exposed to subsonic transverse crossflow. Linear stability analysis was utilized to describe the characteristic length scale and instability growth rate of the surface wave. Empirical relation obtained by other researchers has been used to verify the validness of the present model which shows great consistency in the surface breakup region. In the column breakup region, it has a large discrepancy because of its strong nonlinear effect near the breakup point. 


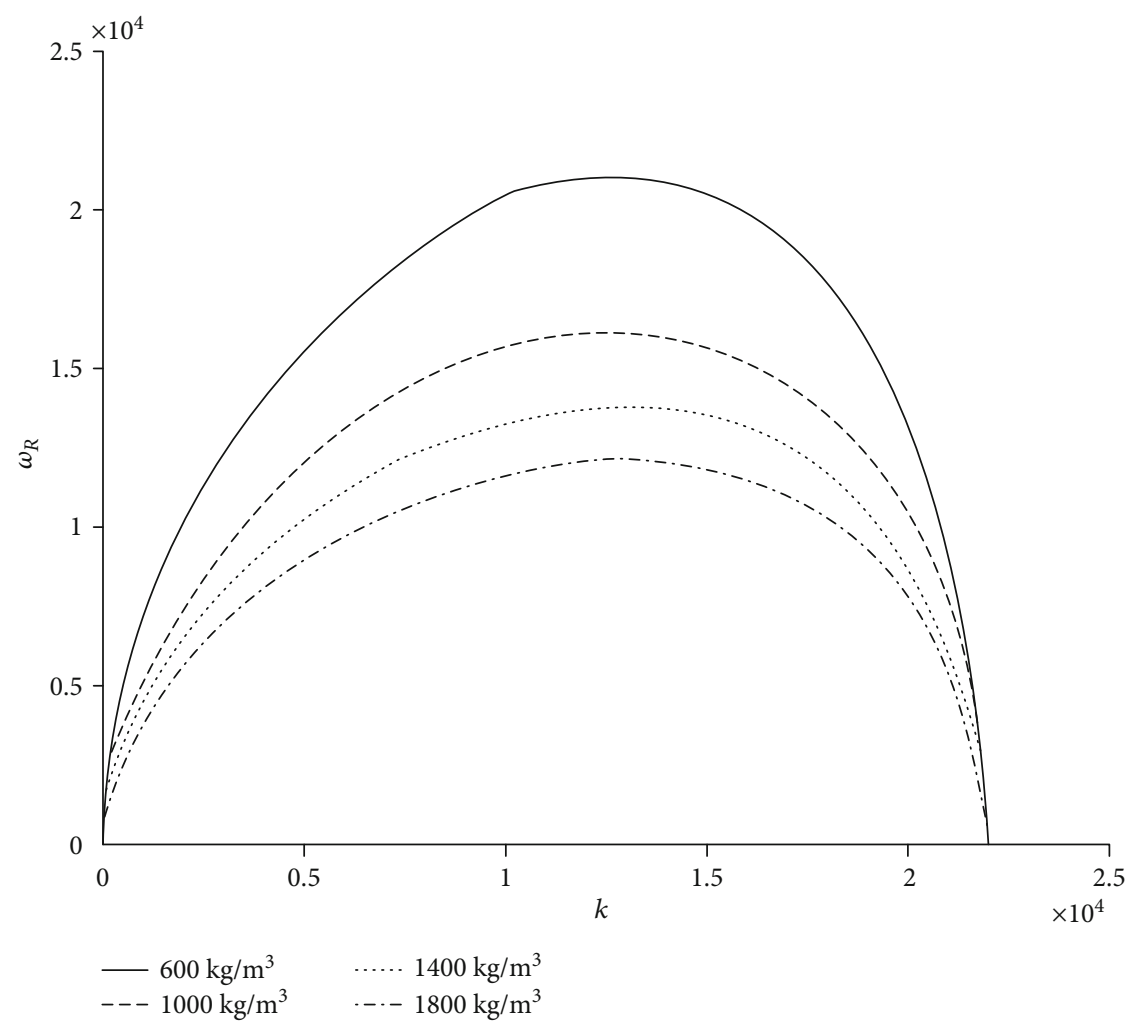

(a)

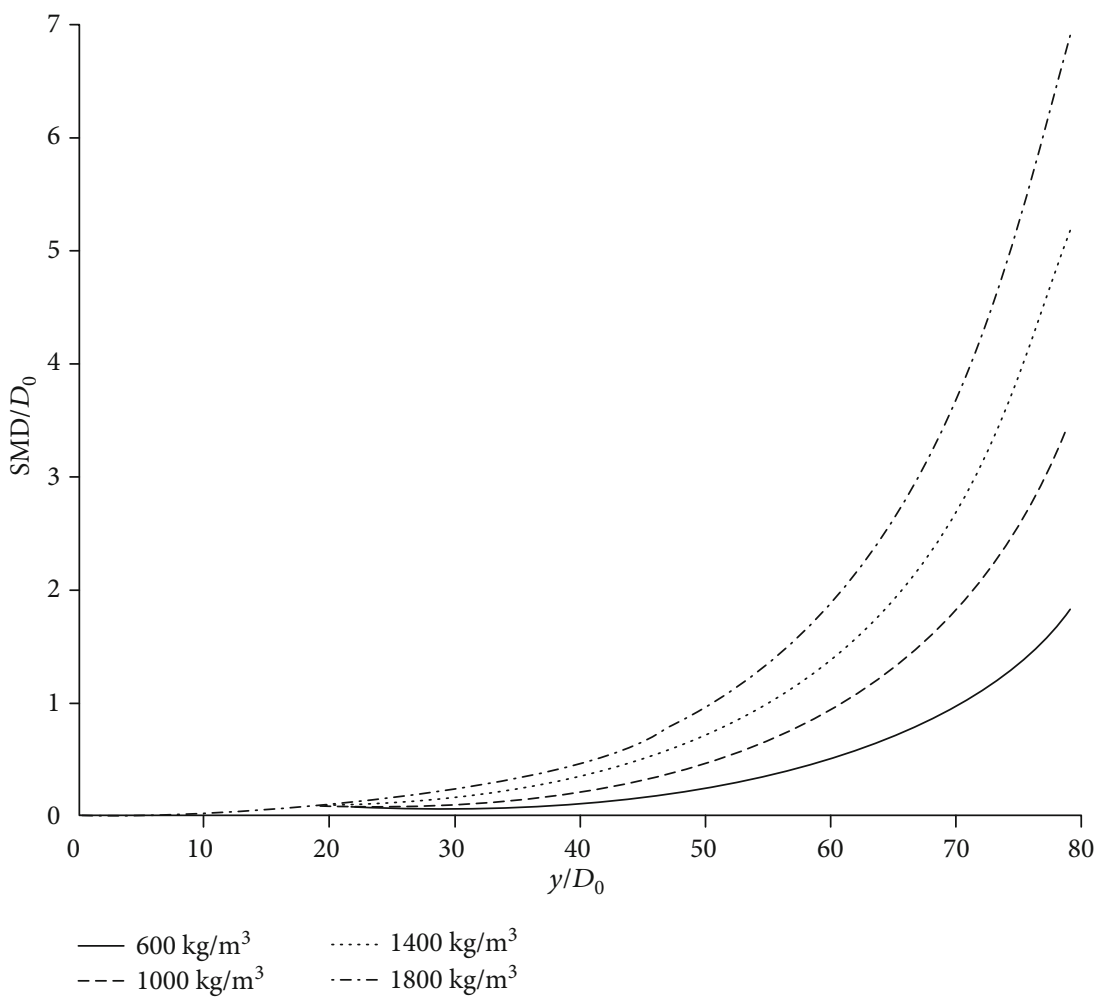

(b)

FiguRE 5: Effect of liquid density to (a) surface wave growth rate and (b) droplet size distribution $\left(\sigma=0.0728 \mathrm{~N} / \mathrm{m}^{2}, \rho_{G}=1.29 \mathrm{~kg} / \mathrm{m}^{3}, u_{L}=\right.$ $13.39 \mathrm{~m} / \mathrm{s}$, and $q=96.35$ ). 


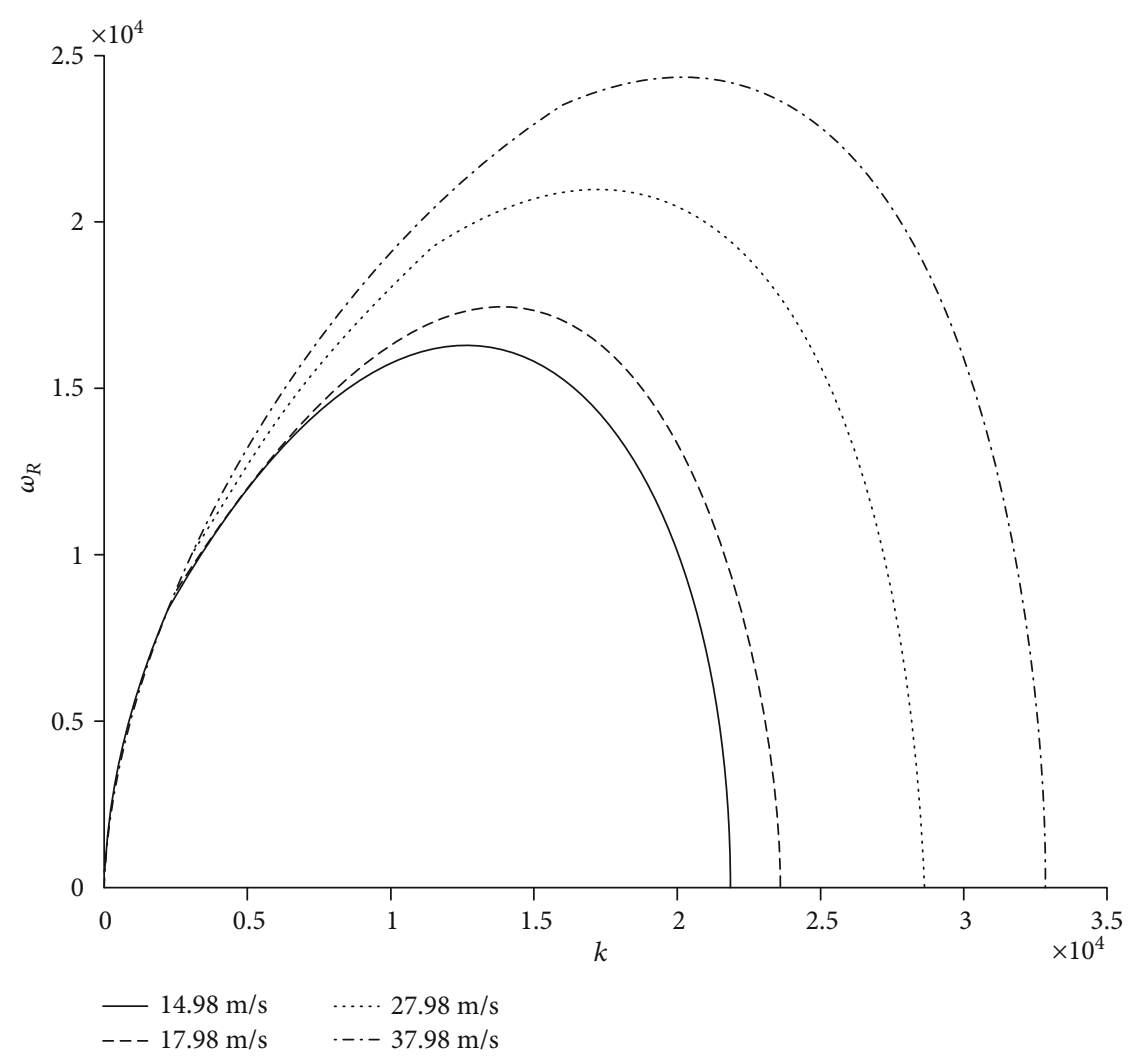

(a)

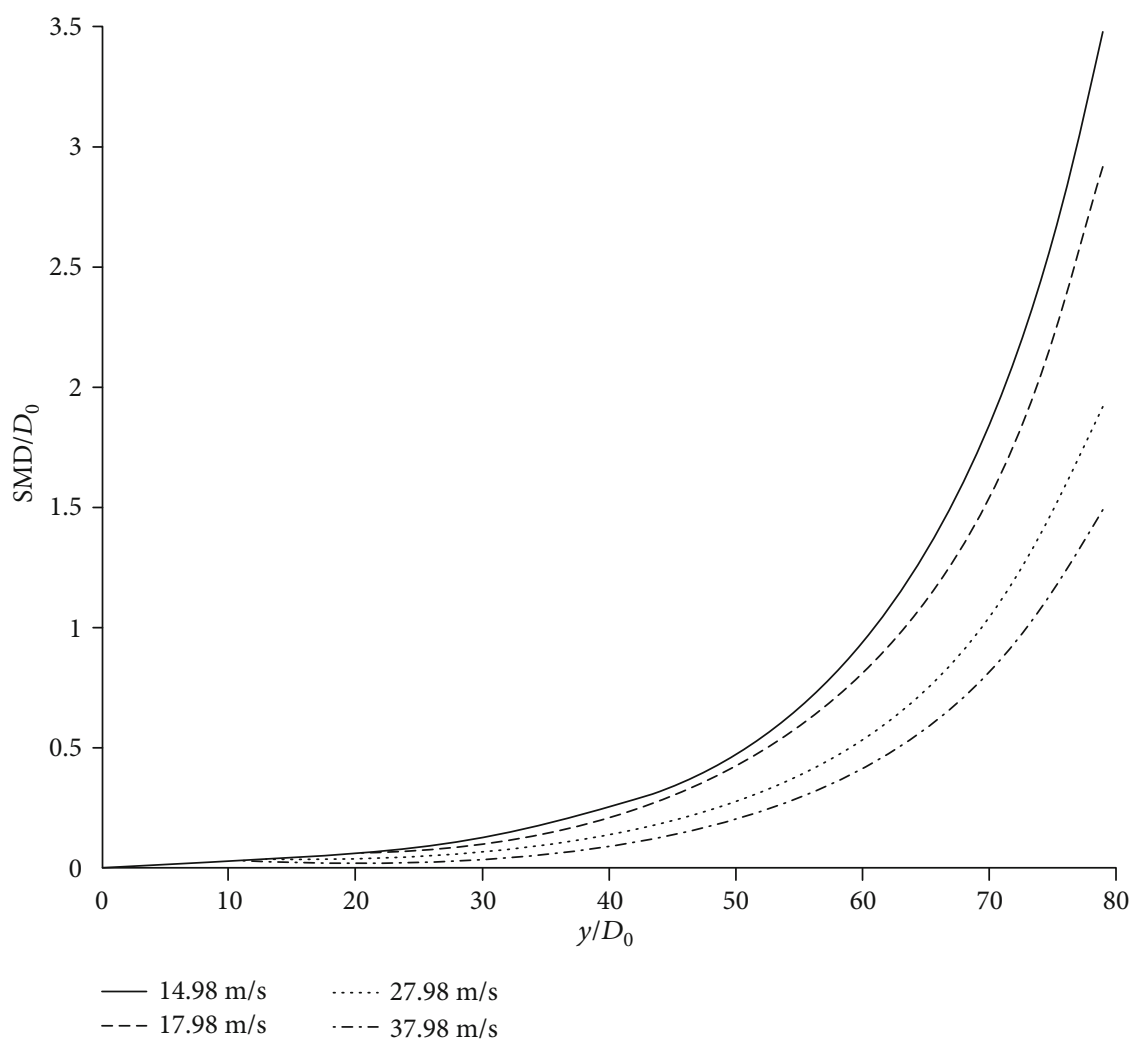

(b)

Figure 6: Effect of transverse gaseous velocity to (a) surface wave growth rate and (b) droplet size distribution $\left(\sigma=0.0728 \mathrm{~N} / \mathrm{m}^{2}, \rho_{L}=\right.$ $1000 \mathrm{~kg} / \mathrm{m}^{3}, \rho_{G}=1.29 \mathrm{~kg} / \mathrm{m}^{3}$, and $\left.q=96.35\right)$. 


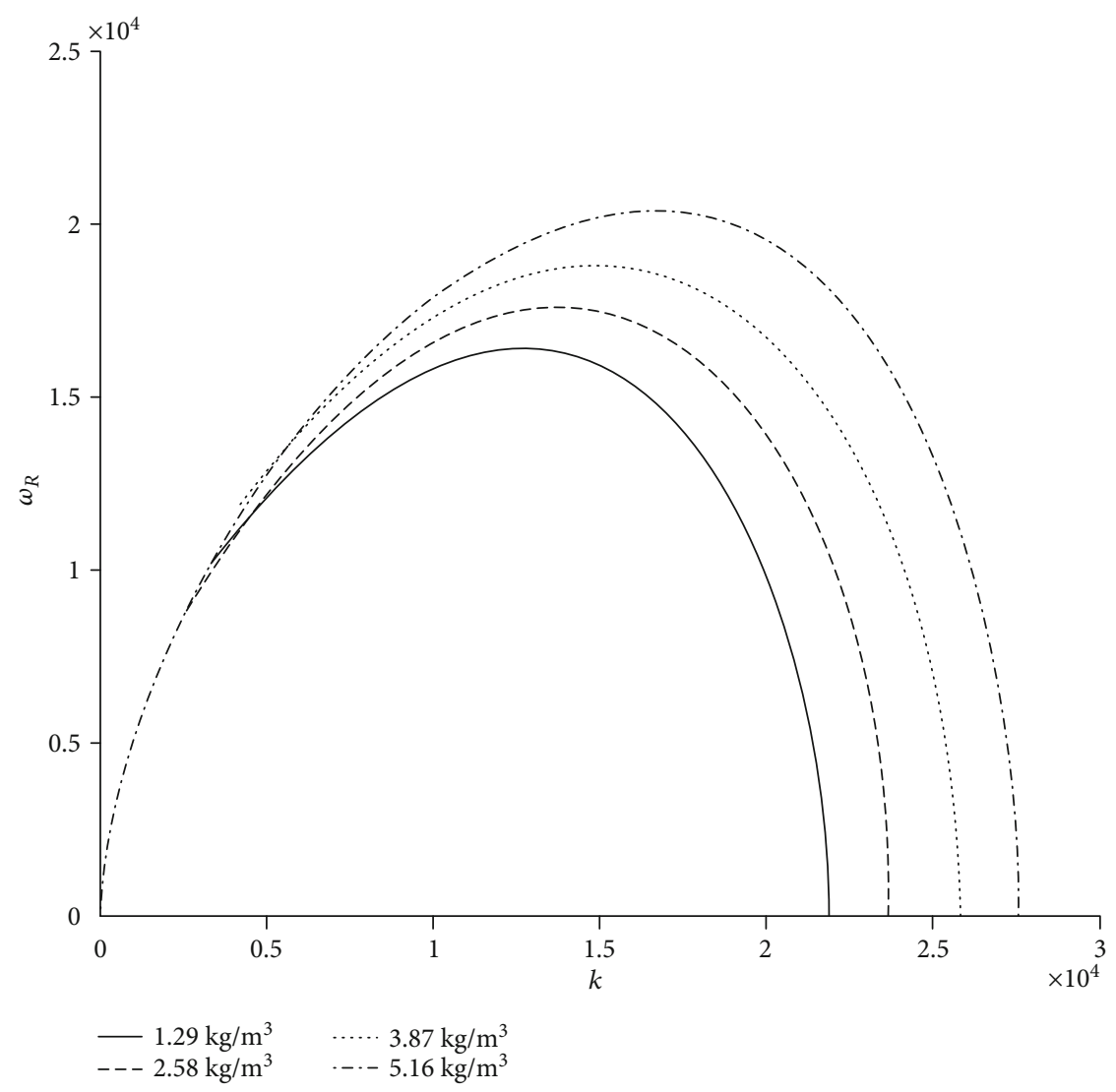

(a)

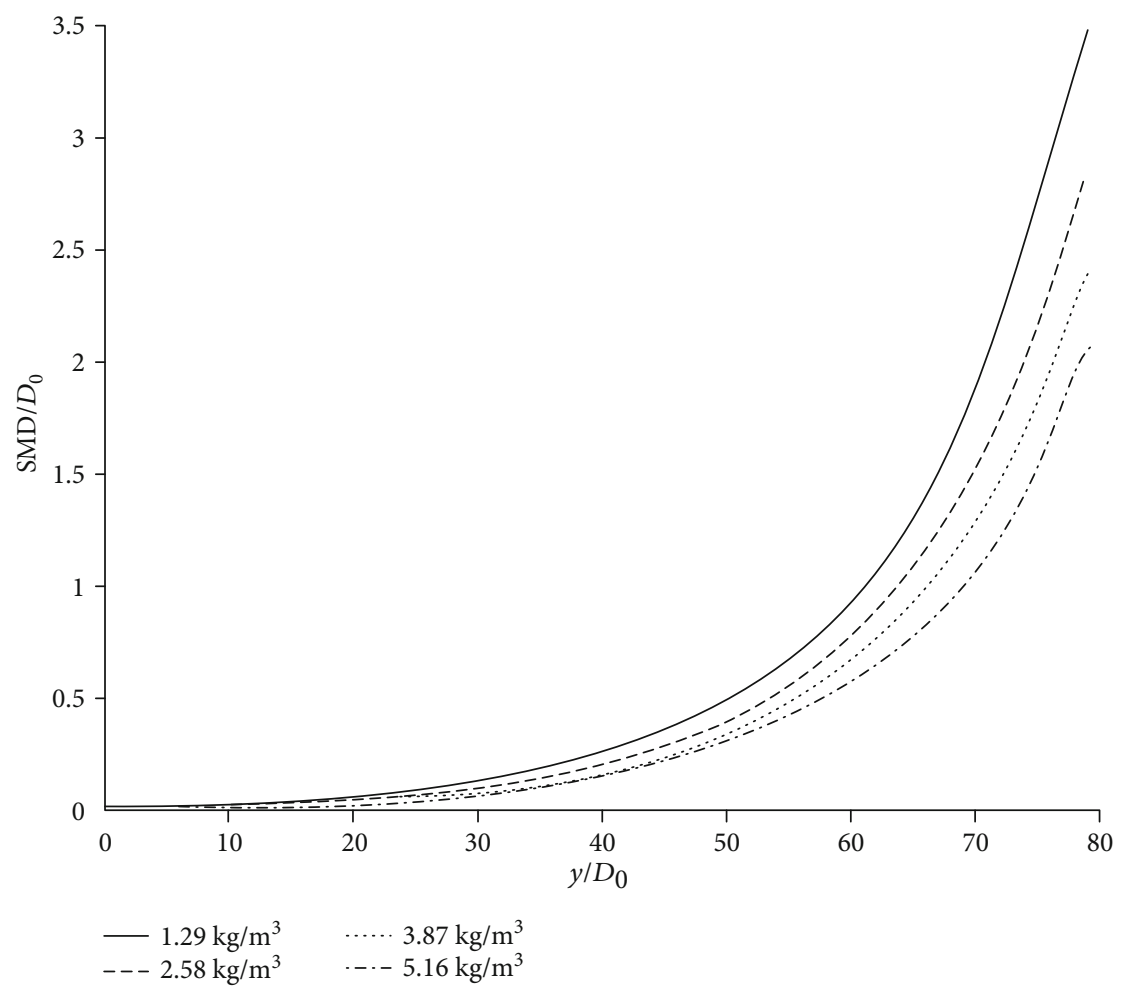

(b)

Figure 7: Effect of transverse gaseous density to (a) surface wave growth rate and (b) droplet size distribution $\left(\sigma=0.0728 \mathrm{~N} / \mathrm{m}^{2}, \rho_{L}=\right.$ $1000 \mathrm{~kg} / \mathrm{m}^{3}, u_{L}=13.39 \mathrm{~m} / \mathrm{s}$, and $\left.q=96.35\right)$. 


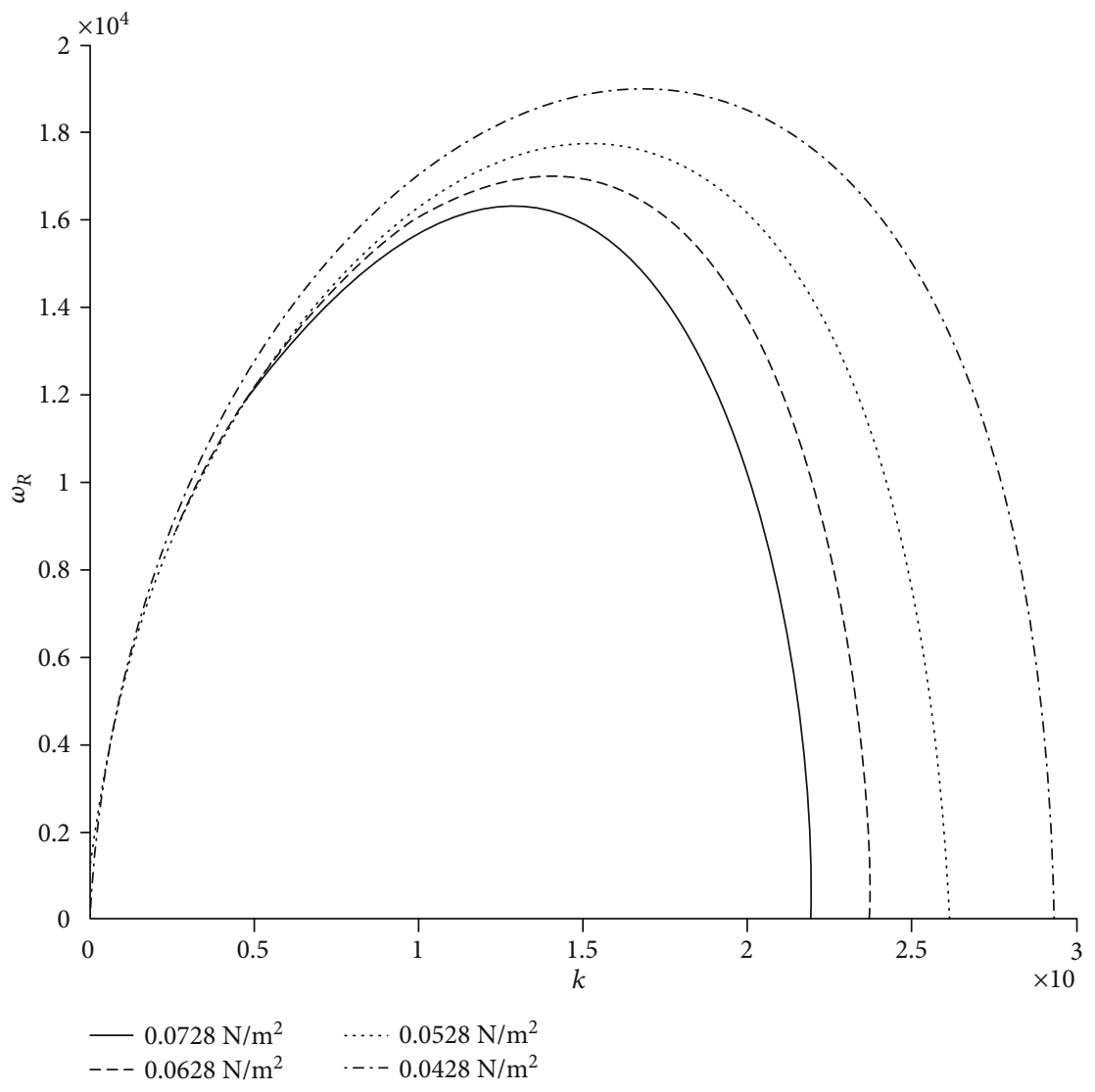

(a)

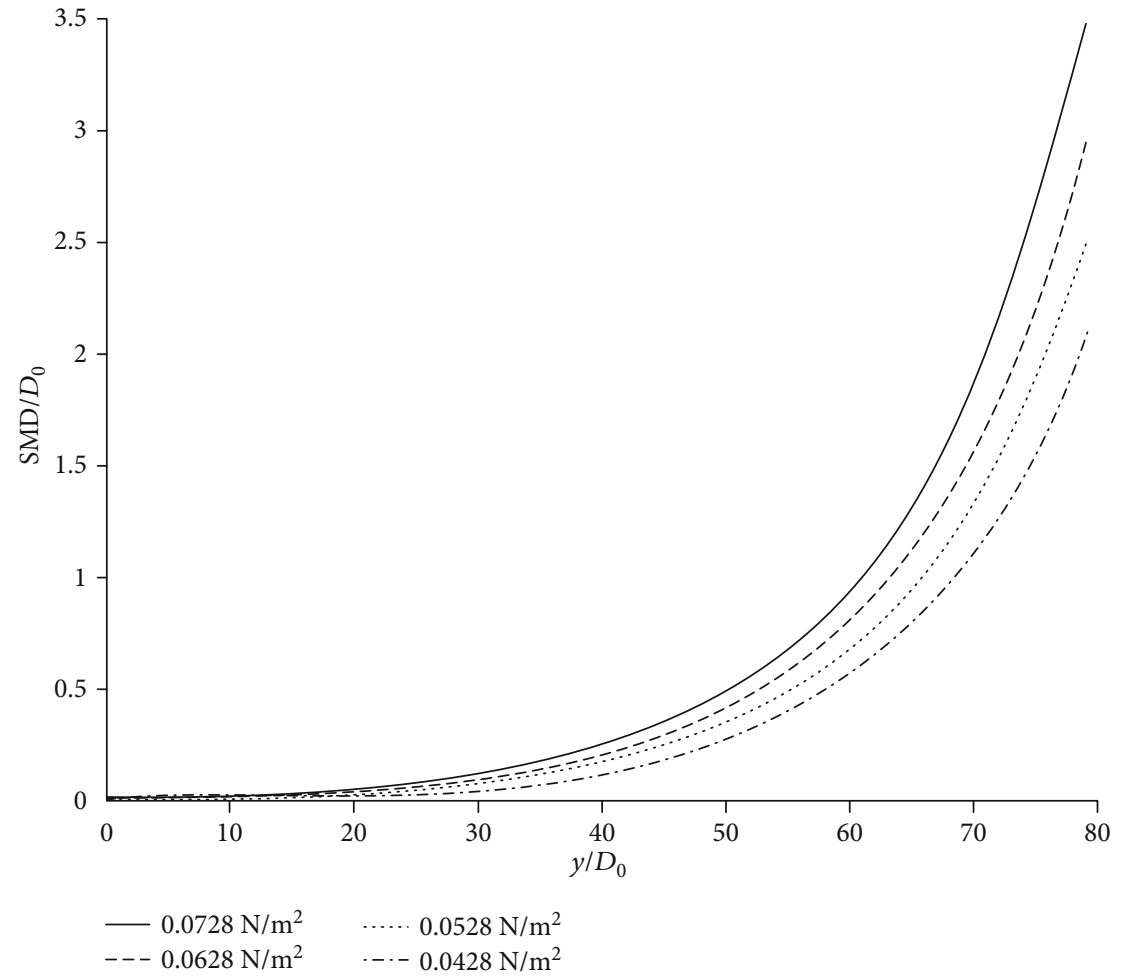

(b)

FiguRE 8: Effect of surface tension to (a) surface wave growth rate and (b) droplet size distribution $\left(\rho_{g}=1.29 \mathrm{~kg} / \mathrm{m}^{3}, \rho_{L}=1000 \mathrm{~kg} / \mathrm{m}^{3}, u_{G}\right.$ $=37.98 \mathrm{~m} / \mathrm{s}, u_{L}=13.39 \mathrm{~m} / \mathrm{s}$, and $\left.q=96.35\right)$. 
The effects of various flow parameters on the maximum growth rate and droplet size spatial distribution have been investigated. At the same height of the liquid column, it will generate more tiny droplets near the exit of the injector with the increase of injection velocity, transverse gaseous velocity, and liquid density. Pressurized transverse gaseous flow promotes surface breakup that smaller droplets appear at the onset of the liquid column. However, increasing surface tension makes ligaments and droplets more difficult to shed from the liquid jet and generates more large droplets.

In the future, the present model can be modified to satisfy characteristics in the column breakup region and related results can be extended to high pressure and temperature environment. In addition, we can consider the effect of heat and mass transfer on the breakup process of liquid jets and droplet size spatial distribution in transverse gaseous flow, which is closer to real working conditions in the thrust engine.

\section{Data Availability}

The data used to support the findings of this study are available from the corresponding author upon request.

\section{Conflicts of Interest}

The authors declare that there is no conflict of interest regarding the publication of this paper.

\section{Acknowledgments}

This research was supported by the National Natural Science Foundation of China (grant Nos. 11872091, 11672025, and U1837211) and the National Natural Science Funds for Distinguished Young Scholars (grant No. 11525207).

\section{References}

[1] H. Wang, Z. Wang, M. Sun, and H. Wu, "Combustion modes of hydrogen jet combustion in a cavity-based supersonic combustor," International Journal of Hydrogen Energy, vol. 38, no. 27, pp. 12078-12089, 2013.

[2] W. Huang and L. Yan, "Numerical investigation on the ramscram transition mechanism in a strut-based dual-mode scramjet combustor," International Journal of Hydrogen Energy, vol. 41, no. 8, pp. 4799-4807, 2016.

[3] M. Sun, Z. Zhong, J. Liang, and H. Wang, "Experimental investigation on combustion performance of cavity-strut injection of supercritical kerosene in supersonic model combustor," Acta Astronautica, vol. 127, pp. 112-119, 2016.

[4] H. Wang, Z. Wang, M. Sun, and N. Qin, "Large eddy simulation of a hydrogen-fueled scramjet combustor with dual cavity," Acta Astronautica, vol. 108, pp. 119-128, 2015.

[5] Z. Wang, M. Sun, H. Wang, J. Yu, J. Liang, and F. Zhuang, "Mixing-related low frequency oscillation of combustion in an ethylene-fueled supersonic combustor," Proceedings of the Combustion Institute, vol. 35, no. 2, pp. 2137-2144, 2015.

[6] M. B. Sun, Z. G. Wang, J. H. Liang, and H. Geng, "Flame characteristics in supersonic combustor with hydrogen injection upstream of cavity flameholder," Journal of Propulsion and Power, vol. 24, no. 4, pp. 688-696, 2008.

[7] M. A. Kolpin, K. P. Horn, and R. E. Reichenbach, "Study of penetration of a liquid injectant into a supersonic flow," AIAA Journal, vol. 6, no. 5, pp. 853-858, 1968.

[8] N. Nejad, A. Schetz, and A. Jakubowski, "Mean droplet diameter resulting from atmoization of a transverse liquid jet in a supersonic airstream," in International Meeting and Technical Display on Global Technology 2000, pp. 1-16, USA, May 1980.

[9] F. Xiao, Z. G. Wang, M. B. Sun, J. H. Liang, and N. Liu, "Large eddy simulation of liquid jet primary breakup in supersonic air crossflow," International Journal of Multiphase Flow, vol. 87, pp. 229-240, 2016.

[10] P. Li, Z. Wang, M. Sun, and H. Wang, "Numerical simulation of the gas-liquid interaction of a liquid jet in supersonic crossflow," Acta Astronautica, vol. 134, pp. 333-344, 2017.

[11] L. Y. Wu, Breakup and atomization mechanism of liquid jet in supersonic crossflow, Doctoral degree paper, National University of Defense Technology, Changsha, 2016.

[12] J. Becker and C. Hassa, "Breakup and atomization of a kerosene jet in CROSSFLOWAT elevated pressure," Atomization and Sprays, vol. 12, no. 1-3, pp. 49-68, 2002.

[13] G. Vich and M. Ledoux, "Investigation of a liquid jet in a subsonic cross-flow," International Journal of Fluid Mechanics Research, vol. 24, no. 1-3, pp. 1-12, 1997.

[14] P. K. Wu, K. A. Kirkendall, R. P. Fuller, and A. S. Nejad, "Breakup processes of liquid jets in subsonic crossflows," Journal of Propulsion and Power, vol. 13, no. 1, pp. 64-73, 1997.

[15] K. A. Sallam, C. Aalburg, and G. M. Faeth, "Breakup of round nonturbulent liquid jets in gaseous crossflow," AIAA Journal, vol. 42, no. 12, pp. 2529-2540, 2004.

[16] S. Rezaei, F. Vashahi, G. Ryu, and J. Lee, "On the correlation of the primary breakup length with fuel temperature in pressure swirl nozzle," Fuel, vol. 258, pp. 1-10, 2019.

[17] A. Amighi and N. Ashgriz, "Trajectory of a liquid jet in a high temperature and pressure gaseous cross flow," Journal Engineering Gas Turbine and Power, vol. 141, no. 1, pp. 1-11, 2019.

[18] A. Amighi and N. Ashgriz, "Global droplet size in liquid jet in a high-temperature and high-pressure crossflow," AIAA Journal, vol. 57, no. 3, pp. 1-15, 2019.

[19] M. Broumand and M. Birouk, "Liquid jet in a subsonic gaseous crossflow: recent progress and remaining challenges," Progressive of Energy and Combustion Science, vol. 57, no. 1, pp. 1-29, 2016.

[20] M. Eslamian, A. Amighi, and N. Ashgriz, "Atomization of liquid jet in high-pressure and high-temperature subsonic crossflow," AIAA Journal, vol. 52, no. 7, pp. 1-13, 2014.

[21] C. M. Varga, J. C. Lasheras, and E. J. Hopfinger, "Initial breakup of a small-diameter liquid jet by a high-speed gas stream," Journal of Fluid Mechanics, vol. 497, pp. 405-434, 2003.

[22] S. L. Wang, Y. Huang, and Z. L. Liu, "Theoretical analysis of surface waves on a round liquid jet in a gaseous crossflow," Atomization and Sprays, vol. 24, no. 1, pp. 23-40, 2004.

[23] G. Amini, "Linear stability analysis of a liquid jet in a weak crossflow," Physics of Fluids, vol. 30, no. 8, pp. 1-11, 2018.

[24] E. Mayer, "Theory of liquid atomization in high velocity gas streams," ARS Journal, vol. 31, pp. 1783-1785, 1961.

[25] L. Z. Qin, R. Yi, and L. J. Yang, “Theoretical breakup model in the planar liquid sheets exposed to high-speed gas and droplet 
size prediction," International Journal of Multiphase Flow, vol. 98, pp. 158-167, 2018.

[26] N. Ashgriz and A. Mashayek, Handbook of Atomizaton and Sprays Theory and Application, Springer, New York, 2011.

[27] K. D. Kihm, G. M. Lyn, and S. Y. Son, "Atomization of crossinjecting sprays into convective air stream," Atomization and Sprays, vol. 5, no. 4-5, pp. 417-433, 1995.

[28] S. Tambe, S. Jeng, H. Mongia, and G. Hsiao, "Liquid jets in subsonic crossflow," in 43rd AIAA aerospace sciences meeting and exhibit meeting papers, pp. 1-17, Reno, USA, January 2005.

[29] N. Liu, Z. Wang, M. Sun, R. Deiterding, and H. Wang, "Simulation of liquid jet primary breakup in a supersonic crossflow under adaptive mesh refinement framework," Aerospace Science and Technology, vol. 91, pp. 456-473, 2019.

[30] M. Behzad, N. Ashgriz, and A. Mashayek, "Azimuthal shear instability of a liquid jet injected into a gaseous cross-flow," Journal of Fluid Mechanics, vol. 767, pp. 146-172, 2015.

[31] M. Behzad, N. Ashgriz, and B. W. Karney, "Surface breakup of a non-turbulent liquid jet injected into a high pressure gaseous crossflow," International Journal of Multiphase Flow, vol. 80, no. 1, pp. 100-117, 2006. 